\title{
Problemas na alimentação infantil e sua associação com a doença do refluxo
}

\author{
Problems in children feeding and association with reflux disease
}

\author{
Problemas en la alimentación infantil y su asociacón con la enfermedad por reflujo
}

Aline Curcio de Araújo ${ }^{1 *}$, Alessandra Cabral Granja², Amanda Silva de Oliveira ${ }^{3}$, Ayalla Isadora Santos Gandra $^{3}$, Érica Karina Tiago Ribeiro ${ }^{4}$, Gabriela Fernandes Marques Barbosa ${ }^{5}$, Isabela Maria Arantes ${ }^{6}$, Jacqueline Yamahata Barbosa Shiro ${ }^{7}$, Joslaine Schuartz lachinski ${ }^{8}$, Rhamai Carneiro de Souza9.

\section{RESUMO}

Objetivo: Identificar os problemas na alimentação infantil que apresentam associação com a doença do refluxo gastroesofágico na infância. Revisão bibliográfica: A doença do refluxo gastroesofágico (DRGE) possui causa multifatorial e corresponde a uma disfunção crônica decorrente do fluxo retrógrado de conteúdo gástrico para o esôfago. A exposição frequente do conteúdo gástrico no esôfago pode causar um defeito no esfíncter esofagiano inferior, que desempenha um papel de barreira antirrefluxo. Dessa forma, essa disfunção pode favorecer a ocorrência de relaxamentos transitórios do esfíncter, causando aumento da predisposição à DRGE. Em crianças, o refluxo gastroesofágico se manifesta de forma fisiológica, entretanto, quando concomitante a sintomas diversos e complicações, geralmente decorrentes de problemas na alimentação, poderá causar o desenvolvimento da DRGE, cujas manifestações mais comuns são a pirose e regurgitação, sintomas que podem influenciar negativamente na qualidade de vida da criança e seus familiares. Considerações finais: A DRGE se manifesta de diferentes formas, causando sinais e sintomas muito amplos de acordo com a faixa etária. Sendo assim, é fundamental identificar os grupos de risco, estar atualizado com a diretriz, e fazer orientações quanto à alimentação adequada, a fim de minimizar suas complicações e facilitar o diagnóstico.

Palavras-chave: Refluxo gastroesofágico, Pediatria, Nutrição da criança.

\begin{abstract}
Objective: To identify infant feeding problems associated with gastroesophageal reflux disease in childhood. Bibliographic review: Gastroesophageal reflux disease (GERD) has a multifactorial cause and corresponds to a chronic dysfunction resulting from the retrograde flow of gastric content into the esophagus. Frequent exposure of gastric contents in the esophagus can cause a defect in the lower esophageal sphincter, which acts as an anti-reflux barrier. Therefore, this dysfunction may favor the occurrence of transitory relaxations of this sphincter, causing an increased predisposition to GERD. In children, gastroesophageal reflux manifests itself in a physiological way, however, when concomitant to different symptoms and complications, usually resulting from feeding problems, it can cause the development of GERD, whose most common manifestations are heartburn and regurgitation, symptoms that can influence negatively on the quality of life of the child and his family. Final considerations: GERD manifests itself in different ways, causing very wide signs and symptoms according to the age group. Therefore, it is essential to identify risk groups, be up-to-date with the guidelines, and provide guidance on adequate nutrition in order to minimize their complications and facilitate diagnosis.
\end{abstract}

Keywords: Gastroesophageal reflux, Pediatrics, Child nutrition.

${ }^{1}$ Instituto Tocantinense (ITPAC Porto), Porto Nacional - TO. *E-mail: alinecurcio@hotmail.com

2 Centro Universitário Da Fundação Assis Gurgacz (FAG), Cascavel - PR.

${ }^{3}$ Faculdade de Medicina de Barbacena (FAME), Barbacena - MG.

${ }^{4}$ Instituto Master de Ensino Presidente Antônio Carlos (IMEPAC), Itumbiara - GO.

${ }^{5}$ União das Faculdades dos Grandes Lagos (UNILAGO), São José do Rio Preto - SP.

${ }^{6}$ Universidade de Cuiabá (UNIC), Cuiabá - MT.

7 Universidade Cidade de São Paulo (UNICID), São Paulo - SP.

8 Centro Universitário de Pato Branco (UNIDEP), Pato Branco - PR.

${ }^{9}$ Faculdade de Medicina Estácio de Juazeiro do Norte (FMJ), Juazeiro do Norte - CE. 


\section{RESUMEN}

Objetivo: Identificar problemas en la alimentación infantil asociados con la enfermedad por reflujo gastroesofágico en la infancia. Revisión bibliográfica: La enfermedad por reflujo gastroesofágico (ERGE) tiene una causa multifactorial y corresponde a una disfunción crónica resultante del flujo retrógrado de contenido gástrico hacia el esófago. La exposición frecuente del contenido gástrico en el esófago puede causar un defecto en el esfínter esofágico inferior, que actúa como barrera antirreflujo. Por tanto, esta disfunción puede favorecer la aparición de una relajación transitoria de este esfínter, provocando una mayor predisposición a la ERGE. En los niños, el reflujo gastroesofágico se manifiesta de forma fisiológica, sin embargo, cuando es concomitante a diversos síntomas y complicaciones, generalmente resultantes de problemas de alimentación, puede provocar el desarrollo de ERGE, cuyas manifestaciones más comunes son pirosis y regurgitación, síntomas que pueden influir negativamente sobre la calidad de vida del niño y su familia. Consideraciones finales: La ERGE se manifiesta de diferentes formas, provocando signos y síntomas muy amplios según el grupo de edad. Por tanto, es fundamental identificar los grupos de riesgo, estar al día de la guía y orientar sobre una nutrición adecuada, con el fin de minimizar sus complicaciones y facilitar el diagnóstico.

Palabras clave: Reflujo gastroesofágico, Pediatría, Nutrición del niño.

\section{INTRODUÇÃO}

O refluxo gastroesofágico (RGE) refere-se à passagem retrógrada do conteúdo gástrico para o esôfago, que se integra de gás ou fluido, logo em certas ocasiões pode chegar a atingir a faringe e a boca, podendo ser complementado ou não de regurgitação e/ou vômito, geralmente o refluxo gastroesofágico pode ocorrer de forma natural em lactentes e crianças (DE MESQUITA KSFG, et al., 2018). A proporção em que ele se manifesta, podem acontecer episódios isolados de refluxo que não se caracterizam como uma doença. Devese haver um cuidado maior com indivíduos que manifestam o refluxo gastroesofágico com frequência semanal e com grande intensidade, diante disso o diagnóstico pode resultar na doença do refluxo gastroesofágico (DRGE) (SANTOS JPR, et al., 2020).

A doença do refluxo gastroesofágico (DRGE) é estipulada como uma disfunção do esôfago, com retorno repetido do conteúdo gástrico para o esôfago, ademais individualiza-se por meio de manifestações clínicas podendo ser variadas e inespecíficas, não limitadas absolutamente ao aparelho digestório (SADOVSCKY ADI, et al., 2017). No entanto, a diferenciação entre ambas as situações se faz imprescindível, visto que a conduta médica é distinta para cada uma. Nesse contexto a distinção entre o refluxo gastroesofágico (RGE) e a doença do refluxo gastroesofágico (DRGE) tem de ser feita pela história e exames clínicos minuciosos, que podem vir a determinar se o lactente carece ou não de intervenção terapêutica. Além disso há uma apreensão constante com a excessiva solicitação de exames e prescrições de medicamentos para lactentes saudáveis que apontam regurgitações não ocasionadas pela DRGE (SOARES ACF, et al., 2015).

No entanto a etiopatogenia da DRGE é relativa a diversos fatores, pois a exposição crônica da mucosa esofágica ao conteúdo gastroduodenal é conseguinte do defeito de um ou mais mecanismos de defesa do esôfago, podendo ser por conta da barreira antirrefluxo, dos mecanismos de depuração ou até mesmo dos mecanismos de resistência que estão presentes no epitélio esofágico (CASTELÃO FN, 2018). A seriedade da DRGE resulta da quantidade de conteúdo refluído para dentro do esôfago, além da permanência da secreção em contato com a mucosa, da sensibilidade desta ao refluxo e da propriedade do esôfago de propulsar o suco gástrico de volta para o estômago (ROSEN R, et al., 2018).

Além disso, a RGE é um evento quase global em bebês prematuros, sendo um diagnóstico comum na unidade de terapia intensiva neonatal (EICHENWALD EC, et al., 2018). Nos primeiros seis meses de vida do lactente, os vômitos e regurgitações ocorrem com maior periodicidade, caracterizando como um quadro de refluxo fisiológico (SOARES ACF, et al., 2015). Isso que, o refluxo é mais evidente em crianças do que em adultos, no qual o desenvolvimento fisiológico do esfíncter esofágico faz com que ocorra regurgitamentos mais de uma vez ao dia em crianças e em recém-nascidos (CASTELÃO FN, 2018). Também, as manifestações clínicas são variadas nas faixas etárias, entre lactentes, crianças e adolescentes, com 
sintomas bem diferenciados, as manifestações mais comuns são pirose e regurgitação, no entanto vale salientar que outras doenças, como úlcera péptica, gastrites e até neoplasias podem cursar com um deles (SADOVSCKY ADI, et al., 2017).

Os problemas de alimentação são a principal causa de consultas pediátricas e da gastroenterologia pediátrica, tornando esse tema altamente relevante para o meio acadêmico e profissional na área da saúde, em que, podem refletir em uma má qualidade de vida do indivíduo, devido a precária alimentação/nutrição, afetando no desenvolvimento motor e cognitivo das crianças (VIEIRA SCF,2019). Ademais, a disfunção alimentar pode ou não implicar em uma exata patologia específica, às vezes pode estar relacionado entre outros fatores que levam a esses problemas alimentares, bem como fatores sociais, comportamentais e econômicos, por isso necessita de uma investigação das manifestações apresentadas pelo paciente, os sintomas mais comuns para a contribuição do distúrbio seria ausência de fome durante o horário da refeição, se alimentam rapidamente e/ou as crianças que se alimentam de algo não ideal antes do horário das refeições com isso ocorre a perda do apetite (CHOI YJ, et al., 2016).

Desta forma, o presente estudo tem como objetivo detectar as consequências da alimentação infantil, associando com a doença do refluxo gastroesofágico na infância, visto que o refluxo é comum, decorrente de forma fisiológica no primeiro ano de vida da criança, por conta de múltiplos fatores, sendo estes: imaturidade do sistema digestório, hábitos alimentares e posturais que atrapalham na competência dos mecanismos antirrefluxo favorecendo a desenvolverem a DRGE. Ademais, o estudo identificou uma dificuldade no diagnóstico por conta de um quadro clínico heterogêneo entre as faixas etárias, pois são vastos os sintomas, ocasionando muitas vezes em um subdiagnóstico, provocando um risco à saúde da criança.

\section{REVISÃO BIBLIOGRÁFICA}

\section{Classificação do Refluxo Gastroesofágico}

O refluxo gastroesofágico (RGE) é definido como o retorno involuntário do conteúdo para esôfago, e pode ser dividido em fisiológico ou patológico. O RGE fisiológico é mais comum em crianças de baixa idade, como recém nascidos, e tem como principais sintomas as regurgitações ou também conhecidas como "gorfadas". Em casos de crianças maiores e adultos, pode acontecer no período pós prandial, que seria após se alimentar (FRAGA PL e MARTINS FSC, 2017; DE MATOS NL, 2017).

O RGE patológico, ou doença do refluxo gastroesofágico (DRGE), ocorre quando as regurgitações e vômitos não melhoram após 6 meses de vida, como também podem ocorrer com a falta de resposta ao tratamento medicamentoso e às medidas posturais e, consequentemente manifestam sinais e sintomas de esofagite (FRAGA PL e MARTINS FSC, 2017; DE MATOS NL, 2017).

\section{Epidemiologia e etiologia}

Ainda não há uma evidência quantitativa sobre o RGE infantil na pediatria, uma vez que os dados epidemiológicos, inicialmente, têm por base a história clínica do paciente, abordada apenas pelos sinais e sintomas (SILVA LR, et al., 2017). Cabe aqui ressaltar ainda que quando o RGE gera sintomas e causa transtornos na qualidade de vida do paciente infantil, esse é considerado patológico.

Sendo assim, torna-se notório que a falta de conhecimento sobre o RGE leva ao subdiagnóstico, o que acaba colocando a saúde da criança em risco pela ocorrência de complicações graves. No lactente, porém, o RGE fisiológico é muito frequente, pois trata-se de um distúrbio gastrointestinal transitório que irá depender da maturação do aparelho digestivo no primeiro ano de vida (SADOVSKY ADI, et al., 2017).

Estudos realizados com bebês nos EUA demonstram que, entre 0-3 meses, $50 \%$ dos bebês apresentam pelo menos um episódio de regurgitação por dia; de 4-6 meses, $67 \%$ dos bebês apresentam regurgitação, dos 7-9 meses, a porcentagem cai para $21 \%$ e dos 10-12 meses se observa uma porcentagem mínima de $5 \%$ (PODDAR U, 2019). Dessa forma, é possível observar que as manifestações clínicas costumam ocorrer por volta dos 2 ou 3 meses de idade, havendo um pico aos 4-5 meses e posteriormente uma queda, posto que, é durante o primeiro ano de vida que ocorre a maturação do aparelho digestivo do lactente. 
É válido salientar ainda que, a partir de amplos estudos realizados, há um subgrupo de crianças mais propensos a desenvolver a DRGE, as quais são: crianças com paralisia cerebral; aquelas que necessitam de cirurgia corretiva da atresia esofágica; crianças com hérnia do hiato ou aquelas que tomam alguns fármacos, como os anticolinérgicos, bloqueadores dos canais de cálcio, benzodiazepinas e dopamina (CASTELÃO FN, 2018).

\section{Etiopatogenia e Fisiopatologia}

Anatomicamente, temos uma barreira antirrefluxo formada pelo esfíncter esofágico inferior (EEI), pinçamento diafragmático e ângulo de His que ficam na junção esofagogástrica. Essa barreira exerce a função de impedir o refluxo do conteúdo gástrico para o esôfago. Contudo, quando mecanismos favorecem a ocorrência de relaxamentos transitórios do esfíncter esofagiano inferior (RTEEI) não relacionados com a deglutição, há a predisposição à DRGE. Vale ressaltar que o RTEEl é fisiológico após uma deglutição, sendo um reflexo normal após distensão do estômago, controlado pelo sistema nervoso autônomo. Quando há falha nesse mecanismo de defesa do esôfago, tem-se a DRGE. (DE MATOS NL, 2017; ROSEN R, et al., 2018).

A etiopatogenia da DRGE é multifatorial, mas seu principal fator é a ocorrência de RTEEI. Na DRGE há uma redução da pressão do EEl por mais que 10 (dez) segundos, podendo ser desencadeada por: distensão da luz esofágica, anormalidade na cicatrização epitelial do esôfago, contrações faríngeas, depuração insuficiente e tamponamento do ácido pela salivação, distensão gástrica e esvaziamento gástrico retardado, diminuição dos reflexos neurais de proteção do trato digestivo e sistema respiratório, diminuição do tônus e aumento da pressão abdominal (SADOVSKY ADI, et al., 2017).

A distensão gástrica é o principal estímulo para o RTEEI. Na fisiopatologia do RGE nos lactentes, o volume alimentar ingerido é maior, então predispõe à maior distensão gástrica. Além disso, os lactentes ficam mais tempo em posição horizontal, então a ausência da gravidade favorece o refluxo, assim como a atonia do esfíncter esofágico superior durante o sono, fazendo com que, ocasionalmente, o refluxo atinja as vias aéreas. Além disso, nos lactentes há uma imaturidade dos mecanismos de barreira antirrefluxo, visto que o esfíncter esofágico inferior é curto e de baixa pressão e o ângulo de His e o hiato esofágico são menos eficazes (PIMENTA JR, et al., 2016; GULATI IK e JADCHERLA SR, 2019).

\section{Manifestações clínicas}

As manifestações clínicas do refluxo são diferentes em lactentes, em crianças e em adolescentes. Nos lactentes, os sintomas mais comuns são regurgitações frequentes que podem estar associadas a vômitos, recusa alimentar, diminuição do ganho de peso, alterações no sono, alguns sintomas de disfagia, irritabilidade e apneia (SADOVCKY ADI, et al., 2017). Esses sintomas, como as regurgitações podem ocorrer em qualquer idade, porém o momento que é mais evidente é por volta dos 4 meses, tendo uma redução aproximadamente aos 6 meses e uma melhora significativa quando o paciente tem entre 12 e 15 meses de idade (PIMENTA JR, et al., 2016).

Já em crianças e adolescentes, os sintomas são mais parecidos com os sintomas dos adultos e os mais comuns são dor abdominal de localização epigástrica, pirose retroesternal, náusea matinal, vômitos e eructação excessiva. Além disso, pode haver disfagia, odinofagia e sono agitado. Em ambas as idades, pode haver anemia e perda de peso (SADOVCKY ADI, et al., 2017).

Em crianças maiores, a frequência com que ocorre o avanço para a cronicidade é maior. É comum que exista períodos de recorrência da doença durante anos. Tal fato explica a maior prevalência e maior intensidade das complicações esofágicas da DRGE nessa faixa etária (BURNS DAR, et al., 2017).

A DRGE pode ser associada, ainda, à problemas respiratórios como o estridor intermitente, rouquidão, laringites de repetição, tosse, broncoespasmos, apneia obstrutiva com hipoxemia, bradicardia, cianose, episódios que ameaçam a vida, pneumonias, aspiração, otites de repetição e asma de difícil tratamento. Além disso, é possível existir também alterações neurocomportamentais associadas à DRGE, como irritabilidade, alterações no sono e a síndrome de Sandifer, caracterizada pela presença de postura anormal da cabeça, 
com torcicolo em crianças neurologicamente normais. Logo, é possível perceber que a DRGE pode se manifestar de diferentes formas, causando sinais e sintomas em diferentes sistemas do corpo humano (SILVA LR, et al., 2018).

Vale ressaltar a importância de se observar os sinais de alerta durante a avaliação clínica. Dessa forma é possível identificar precocemente complicações da DRGE e, então, descartar doenças concomitantes que podem apresentar os mesmo sinais e sintomas da DRGE. Portanto, deve-se prestar atenção aos seguintes sintomas: irritabilidade, recusa de alimentação, postura distônica do pescoço, regurgitação recorrente com ou sem vômito, azia, dor no peito, dor epigástrica, hematêmese, disfagia, odinofagia, respiração ofegante, estridor, tosse e rouquidão (ROSEN R, et al., 2018).

\section{Prevenção}

Crianças com hábitos alimentares irregulares como a preferência por lanches e hábitos alimentares noturnos, intervalo reduzido entre o jantar e o horário de dormir e preferência por alimentos líquidos estão mais propensas a desenvolver a DRGE. Sendo assim, é indicado na prevenção do refluxo que a criança tenha uma alimentação adequada, evitando excesso de alimentos líquidos e que faça um intervalo significativo entre o jantar e o momento de ir dormir (CHOI YJ, et al., 2016).

Em lactentes uma forma de prevenir a doença do refluxo é através da remoção de alguns alimentos da dieta materna, como os ovos e o leite de vaca. Somado a isso, quando acordados, é recomendado que os bebês sejam colocados em decúbito prono ou lateral esquerdo para diminuir os episódios de refluxo (BORODINA G e MOROZOV S, 2020).

\section{Diagnóstico}

A detecção do refluxo do conteúdo gástrico para o estômago não necessariamente significa que o paciente tenha DRGE (BURNS DAR, et al., 2017). Por isso o diagnóstico começa a partir da história clínica do paciente. Em crianças de baixa idade, casos de regurgitações sem nenhum outro achado indica RGE fisiológico. No caso de outras manifestações como choro constante, irritabilidade, broncoespasmo persistente, entre outros, caracteriza a presença de refluxo gastroesofágico patológico. A anamnese detalhada e um exame físico cuidadoso podem auxiliar na identificação do paciente com DRGE, porém, nesses casos, exames como endoscopia e sonda de pH são indispensáveis para confirmação (BAIRD DC, et al., 2015; DE MATOS NL, 2017).

Os métodos de exame de diagnóstico devem ser analisados cuidadosamente, compreendendo as limitações e capacidades de cada um e evitando submeter os pacientes, principalmente crianças, a testes muito invasivos, caros e inapropriados. Por exemplo, a radiografia contrastada de esôfago, estômago e duodeno (RxEED) é um exame de baixo custo e fácil execução, porém não é adequada para diagnóstico da doença do refluxo, pois não tem a capacidade de quantificar os episódios de refluxo, bem como a ultrassonografia esofagogástrica não é recomendada, sendo mais importante no diagnóstico diferencial de estenose hipertrófica de piloro (SILVA LR, et al., 2018; BURNS DAR, et al., 2017).

Existem também a manometria esofágica, a qual avalia a motilidade do esôfago, sendo mais indicado para pacientes que apresentam disfagia e odinofagia. Como também o teste terapêutico empírico com supressão ácida, mas são indicados apenas para crianças maiores e adolescentes (BURNS DAR, et al., 2017).

Os exames mais indicados e adequados são a endoscopia digestiva alta com biópsia, que é indicada em casos de sintomas persistentes e complicações da DRGE, e a pHmetria esofágica, em casos que a endoscopia estiver normal e os sintomas persistirem mesmo com uso de inibidores de bomba de prótons. A impedanciometria esofágica intraluminal é um novo método que também é eficaz, podendo ter mais valor que a pHmetria ao monitorar a quantidade e qualidade do material refluído (SILVA LR, et al., 2018; BURNS DAR, et al., 2017).

Na maioria dos casos de pacientes pediátricos, como lactentes e crianças, o uso de exames de imagem não é recomendado dependendo da gravidade e do conjunto de sintomas. Porém, em casos de condições 
clínicas associadas que fazem com que a prevalência da doença aumente como a asma, fibrose cística, disfunções respiratórias e tosse crônica, a realização desses exames pode ser indicada (SADOVSCKY ADI, et al., 2017). Assim, os testes diagnósticos são realizados para diferenciar o refluxo fisiológico e patológico, como também espera-se encontrar a presença de RGE ou de suas complicações para assim estabelecer qual tratamento deve ser realizado para maior eficácia ao paciente (PIMENTA JR, et al., 2016; BURNS DAR, et al., 2017).

\section{Tratamento}

O tratamento para DRGE deve ser analisado de forma específica para cada caso e idade. Em bebês, a regurgitação tende a decrescer até o 12 mês de vida na maioria dos casos. Logo, o tratamento baseia-se em mudar a posição da criança quando está acordada, diminuir o volume de alimentação oferecida ou até mesmo considerar o uso de espessantes. Já em bebês que ainda são amamentados, a indicação é a retirada do leite e ovo da dieta da mãe, uma vez que esses alimentos estão muito relacionados à ocorrência dos sintomas (BAIRD DC, et al., 2015).

Como a postura também interfere na diminuição dos sinais e sintomas, recomenda-se que o lactente pós mamada fique na posição vertical de 20 a 30 minutos e, na hora do sono, é indicada posição dorsal com elevação da cabeça. (SADOVCKY ADI, et al., 2017). Também é indicado não usar roupas apertadas e trocar as fraldas antes de amamentar, como também evitar a exposição ao tabaco, pois induz o relaxamento do EEI e também pode ser prejudicial para a saúde respiratória da criança (BURNS DAR, et al., 2017).

Em crianças e adolescentes a mudança no estilo de vida também é necessária e recomenda-se a melhora nos hábitos alimentares e consequente perda de peso, evitar se alimentar muito tarde, elevar a cabeça ao dormir e evitar o uso de álcool e fumar. $\mathrm{O}$ uso de terapias médicas como os antagonistas do receptor $\mathrm{H}_{2}$ da histamina e inibidores da bomba de prótons também são indicados. O tratamento cirúrgico é indicado apenas em casos em que a terapia médica não foi bem sucedida (BAIRD DC, et al., 2015).

Quanto aos medicamentos utilizados, o uso de antagonistas do receptor de histamina, possibilita maior melhora em relação ao vômito e regurgitação (FERREIRA CT, et al., 2014). Já o uso de domperidona também é indicado, porém apenas em alguns casos, e tem como consequente à melhora do vômito, quando associado a um antiácido, como alginato, há chances de diminuir os sintomas de DRGE, pois a domperidona é um antidopaminérgico que facilita o esvaziamento gástrico (ROSEN R, et al., 2018).

Por sua vez, o uso de medicamentos inibidores da bomba de prótons (IBP) como lansoprazol, esomeprazol, rabeprazol, pantoprazol e omeprazol é mais indicado para tratamento de bebês com irritabilidade e choro (ROSEN R, et al., 2018). Os IBP também são indicados em casos de esofagite erosiva, estenose péptica ou esôfago de Barret, bem como em crianças que precisam de um bloqueio mais eficaz da secreção ácida, pois esse fármaco mantém o pH gástrico acima de 4 por tempos maiores, além de inibir a secreção ácida provocada pelos alimentos (SILVA LR, et al., 2018).

Outras mudanças indicadas nos hábitos alimentares é a diminuição de ingestão de bebidas gaseificadas e cafeinadas e de alimentos muito gordurosos, como frituras. Esses alimentos tendem a diminuir a pressão no esfíncter esofágico inferior e o tempo de esvaziamento gástrico, o que pode ocasionar piora dos sintomas da DRGE (SADOVCKY ADI, et al., 2017).

\section{CONSIDERAÇÕES FINAIS}

A presente revisão evidenciou que o refluxo é comum e ocorre de forma fisiológica nas crianças, especialmente no $1^{\circ}$ ano de vida, devido à imaturidade funcional do sistema digestório, hábitos alimentares e posturais que prejudicam a competência dos mecanismos antirrefluxo, deixando-as mais propensas a desenvolverem a DRGE. Constatou-se que há dificuldade no diagnóstico, visto que há um quadro clínico heterogêneo entre as faixas etárias, pois os problemas na alimentação causados pela DRGE podem ser desde pirose, vômitos, alterações no sono e regurgitação até úlceras e perda de peso excessiva, o que pode acarretar em um subdiagnóstico, podendo assim colocar a saúde da criança em risco. 


\section{REFERÊNCIAS}

1. BAIRD DC, et al. Diagnosis and Treatment of Gastroesophageal Reflux in Infants and Children. American family physician, 2015; 92(8): 705-714.

2. BORODINA G, MOROZOV S. Children with gastroesophageal reflux disease consume more calories and fat compared to controls of the same weight and age. Journal of Pediatric Gastroenterology and Nutrition, 2020; 70(6): 808-814.

3. BURNS DAR, et al. Tratado de Pediatria: Sociedade Brasileira de Pediatria, Volume 1; São Paulo: Manole; $2017 ; 712$ p.

4. CASTELÃO FN. Doença do refluxo gastroesofágico em idade pediátrica. Dissertação (Mestrado Integrado em Medicina) - Faculdade de Medicina. Universidade de Lisboa, Lisboa, 2018; 27p.

5. $\mathrm{CHOI}$ YJ, et al. Dietary habits and gastroesophageal reflux disease in preschool children. Korean journal of pediatrics. 2016; 59(7): 303- 307.

6. DE MATOS NL. Refluxo gastroesofágico no primeiro ano de vida: fisiológico ou patológico? Dissertação (Especialização em Gastroenterologia Pediátrica) - Faculdade de Medicina. Universidade Federal de Minas Gerais, Belo Horizonte, 2017; 44p.

7. DE MESQUITA KSFG, et al. Manifestações orais da doença da doença do refluxo gastroesofágico em crianças. Revista Uningá, 2018; 55(2): 14-23.

8. EICHENWALD EC. et al. Diagnosis and management of gastroesophageal reflux in preterm infants. Pediatrics, 2018; 142(1): 1-11.

9. FERREIRA CT et al. Doença do refluxo gastroesofágico: exageros, evidências e a prática clínica. Jornal Pediátrico, 2014; 90(2): 105-117.

10. FRAGA PL, MARTINS FSC. Doença do Refluxo Gastroesofágico: uma revisão de literatura. Cadernos UniFOA, 2017; 18(7): 93-99.

11. GULATI IK, JADCHERLA SR. Gastroesophageal Reflux Disease in the Neonatal Intensive Care Unit Infant: Who Needs to Be Treated and What Approach Is Beneficial?. Pediatr Clin North Am. 2019; 66(2): 461-473.

12. NORTON RC, PENNA FJ. Refluxo gastroesofágico. Jornal de pediatria, 2000; 76(2): 1-7.

13. PIMENTA JR, et al. Refluxo gastroesofágico. Revista. Médica de Minas Gerais, 2016; 26(6): 76-81.

14. PODDAR U. Gastroesophageal reflux disease (GERD) in children. Paediatrics and international child health, 2019; $39(1): 7-12$.

15. ROSEN R, et al. Pediatric gastroesophageal reflux clinical practice guidelines. Journal Pediatric Gastroenterology Nutrition, 2018; 66(1): 516-554.

16. SADOVSKY ADI, et al. Regurgitação do lactente (Refluxo Gastroesofágico Fisiológico) e doença do refluxo gastroesofágico em Pediatria. Sociedade Brasileira de Pediatria - Documento Científico - Departamento de Gastroenterologia Pediátrica, 2017.

17. SANTOS JPR, et al. Doença do Refluxo Gastroesofágico em Lactentes: análise global / gastroesophageal reflux disease in infants. Brazilian Journal Of Health Review, 2020; 3(5): 14663-14677

18. SILVA LR, et al. Manual de Residência em Gastroenterologia Pediátrica, 2018; 586 p.

19. SOARES ACF, et al. Conhecimento e prática de pediatras brasileiros sobre a doença do refluxo gastroesofágico em lactentes. Revista Paulista de Pediatria, 2015; 33(1): 12-18.

20. VIEIRA SCF. Adesão de pediatras às diretrizes para doenças gastrointestinais. Tese (Doutorado em Ciências da Saúde) - Universidade Federal de Sergipe, Sergipe, 2019; 122 p. 